\title{
NOUVEAUX COMMENTAIRES DES TEXTES DE QUELQUES VASES POLYCHROMES DE LA PERIODE CLASSIQUE MAYA
}

Michel Davoust

\section{Introduction}

Un vase polychrome Maya présente un ou plusieurs vases de caractère mythique ou historique comportant un texte glyphique. D'après Coe 1973, le texte glyphique comprend en général deux parties: une phrase principale "phrase standart" horizontale répétée dans de nombreux vases, tandis que les phrases secondaires verticales sont spécifiques de chaque vase et en rapport avec les personnages de ces scènes. Dans l'étude de 3 vases polychromes: Vases Princeton 14 et 19 et vase Grolier 47 publiés par Coe 1973 et 1978 , nous allons montrer les nombreux problèmes de lecture posés par ces textes de céramique. Il est d'abord difficile de localiser et de dater ces vases de la période classique. Ces glyphes de style cursif comportent parfois des affixes ou des éléments principaux non transcrits dans le catalogue de Thompson. De plus l'analyse iconique de ces scènes a été partiellement faite. Nous avons fait appel a un plus large corpus de vases, à l'utilisation des textes des monuments et des Codex. Pour les lectures phonétiques nous avons utilisé principalement les travaux de l'école nord américaine notamment les participants de la conférence d'Albany 1979 (publiée en 1984 par Justeson et Campbell) et ceux plus réçent de Bricker 1986, Grube 1986 et Stuart 1987. Ainsi que notre étude de 1987 dressant le bilan du déchiffrement de l'écriture Maya. 


\section{Méthode}

Localisation et datation de ces 3 vases

Le texte du vase Grolier 47, comporte les glyphes emblèmes de Naranjo et Yaxha. Ce vase serait donc originaire de Naranjo. Le vase Princeton 14 comporte les glyphes emblèmes de $\mathrm{Ca}$ lakmul, Tikal et Machaquila. Or Tikal est a égale distance de Calakmul au nord et de Machaquila au sud. Ce vase pourrait être également de la région de Naranjo, confirmé par Coe 1978 et Reents 1987. D'après ce dernier auteur l'analyse des composants chimiques révèlent des éléments identiques dans les vases Grolier 47, Princeton 14 et des vases découverts à Naranjo. De plus l'étude stylistique confirme cette hypothèse. Par contre le vase Princeton 19 semble originaire de la région de Nebaj dans les Hautes Terres du Guatemala d'après Coe 1978. Ces vases datent de la période classique moyenne ou rèçente d'après Coe 1973 et 1978. Le vase Grolier 47 porte le nom du chef IIIx de Naranjo, or il est indiqué sur la stèle 19 de ce site daté 9.17.0.0.0. et le glyphe de son épouse est indiqué sur la stèle 8 datée 9.18 .10 .0 .0 .

Le vase Princeton 19 peut être contemporain du vase Grolier 26 daté 10.0.0.0. 7 Ahau (18 Zip) selon Coe 1973 p. 62.

Ces 2 vases portent le nom de chefs identiques et son issus tous les deux de Nebaj dans la vallée du Haut Chixoi dans les Hautes Terres du Guatemala.

\section{Transcription des textes}

Nous avons utilisé le catalogue de Thompson 1962 pour la transcription des textes glyphiques. Dans les textes des vases étudiés le style cursif des glyphes est proche de ceux des Codex et la plupart des affixes et éléments principaux sont retrouvés dans le catalogue de Thompson.

\section{Nouvelles interpretations des glyphes de la phrase standart}

Les travaux réçents de Grube 1986 pour l'ensemble de la phrase standart et les lectures de glyphes particuliers de Houston et Taube 1987 ainsi que Stuart 1987 ont permis une meilleure 
approche de celle-ci. Ainsi Grube 1986 a proposé pour le signe initial (228.617:126) 2 lectures possibles: ila avaitregardé. ali avait dit. et plus réçemment dans une communication personnelle la lecture $C h^{\prime} \circ$ (enfant) pour le glyphe 758:110.

Houston et Taube 1987 ont proposé la valeur: u lac (son plat) et remarqué que ce glyphe n'est rencontré que sur des plats ou des bols. De même le glyphe 61.77.585a. n'est rencontré que dans les textes de vases cylindriques. De son côté Stuart 1987 a proposé 3 lectures nouvelles. D'abord la plus importante $U T z^{\prime} i b$ (son scribe) pour le glyphe $1.248: 585 \mathrm{a}$, puis la lecture: Cacaw (cacao) pour le glyphe 738c.130. et d'après Houston 1987 la lecture: Ahawte (Seigneur arbre) pour le glyphe 168:513.

Nous confirmons d'abord la 2ème lecture: a-li (ya) soit Ali (avait déclaré) pour le glyphe initial: 228.617:126.

Puis nous avons proposé les nouvelles lectures:

$u$-ch'i-bi(1) 61.77:585a. terme Chol pour vase

Ah pay (le guide) pour le glyphe du Dieu N (1014a.17)

tu ut (achevé) pour le glyphe 53.61:565.

Tal Maax (originaire du singe araignée) pour le glyphe 220.755

yich (son visage) pour le glyphe 17.671 .

\section{Analyse structurale du texte des vases}

Nous avons utilisé l'analyse structurale inspirée de celle d'Escalante dans le Codex de Dresde 1971 que nous avions adapté aux textes des monuments (cf. notre étude de 1987 Volume 2: l'analyse structurale des textes des Codex et des monuments). Nous prendrons ici l'exemple du texte du vase Princeton 14. Il comprend 5 phrases cf. Texte 1 . Dans la partie supérieure $\mathrm{du}$ vase nous avons un texte circulaire horizontal et 3 phrases verticales correspondant à chacun des 3 personnages de la scène représentant: Nah Ahaw (le 1er Seigneur) en position de danse. Chacune de ces 3 phrases commencent par un glyphe verbal. De plus le texte horizontal s'articule en 2 phrases commençant chacune par un glyphe verbal en A et J. Dans les 4 cas le glyphe verbal comporte le pronom de la 3ème personne du singulier: $\mathrm{U}$ transcrit par le préfixe 1 . Seul le 1er verbe ne comporte pas 
ce pronom. Nous pouvons donc analyser le texte de la façon suivante:

\section{Phrase composée 1 (Gl.A-I) de type V1-S1-V2-S2-C}

Le premier glyphe en A est le verbe: ali (avait déclaré). Il est suivi du groupe sujet 1 en B-C itzat uch'ibil (l'artisan du vase).

Le deuxième verbe en $\mathrm{D}$ est lu: Ta ut (était) achevé.

Il est suivi du groupe sujet: Nah(Ahaw): Yah Tel Cacaw (premier Seigneur de la fente de l'arbuste cacao).

La phrase se termine par le groupe titre: Chac Ch'oc tal maax (vigoureux enfant provenant du singe araignée).

Donc nous avons une prhase composée de 2 verbes de type: V1-S1-V2-S2-C.

1er verbe (V1) - 1er sujet (S1) - 2ème verbe (V2) - 2ème sujet (S2) - complément (C). D'après Escalante (1971, p. 78), et notre étude de 1987, nous avons dans le Codex de Dresde p. $25 \mathrm{~b}$, une phrase composée de même type: V1-S1-V2-S2-C.

Phrase simple intransitive $2(G l . J$ à $Q$ ) de type $V-S-C$

Cette phrase commence par le glyphe verbal: $U$ Bah (il était allé/premier) en $\mathrm{J}$. Il est suivi d'un glyphe titre difficile à interpréter pour l'instant. En L et M, le groupe sujet est lu: Mah Kina Yahawte (le tout puissant Seigneur de l'arbre).

La phrase s'achève par une série de glyphe titre de $\mathrm{N}$ à $\mathrm{Q}$ :

Ah Chikinil 19 xs (celui de l'ouest)

Ah Ch'inil (celui qui jette)

Chan Cup atnositon (les 4 jicamas)

Ah Bah lahkin (le premier à l'est)

Ici nous avons une phrase simple intransitive de type verbe (V) - groupe sujet (GS) et complèment (C). Nous retrouvons dans le Codex de Dresde le type de phrase simple intransitive: V-S-C, exemple Dresde p. 23 b. 
Phrases simples intransitives (Gl.R1-R5, S1-S5, T1-T5) de type: $V-S-C$

Ces phrases comprennent chacune 5 glyphes et commencent par le même verbe $U$ Bah (il était allé) le groupe endocentrique sujet qui est d'après Escalante un groupe formé d'un glyphe moyen et d'un glyphe satellite comporte dans les 3 cas le même glyphe titre ou attribut: Nah Ahaw (premier seigneur) suivi de 3 glyphes nominaux différents:

\begin{tabular}{l|l} 
Hah Chan & (véritable ciel) \\
Hah Batz' & (véritable singe hurleur) \\
\hline Hah Balam & (véritable jaguar)
\end{tabular}

Les 3 phrases se terminent par un groupe endocentrique locatif. De nouveau nous retrouvons le même glyphe titre: Itzat (habile, astucieux, artisan) dans les 3 groupes. Par contre le glyphe locatif est lu respectivement Can (Serpent) Multun $\mathrm{Zu}$ (la pyramide épaisse) et Balan (caché).

\section{Glyphe nominal: Nah Ahaw dans les vases}

Notre nouvelle lecture de la phrase standart cf. vase Princeton 14 glyphe $\mathrm{A}$ à $\mathrm{I}$, nous permet de voir que celle-ci se référe à Nah Ahaw (premier Seigneur) installé dans l'arbre Cacao et qu'il est le descendant du Singe araignée. Nah Ahaw est l'incarnation du chef Maya défunt dans le monde souterrain. Nous le trouvons associé avec le nom du chef Chan Balam à Palenque Dans le Temple XIV, 3 ans après sa mort le chef Chan Balam est associé avec le glyphe Nah Ahaw dans le texte suivant:

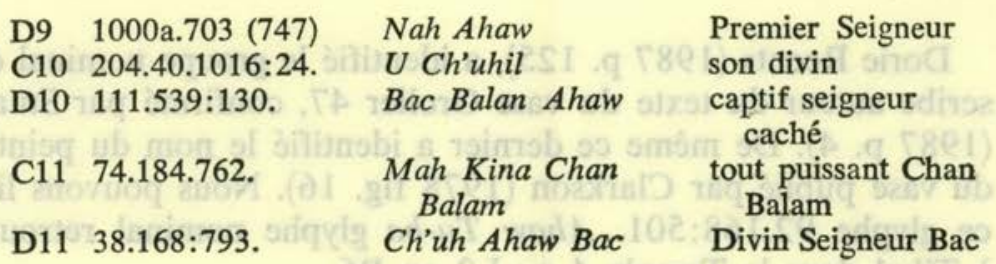


Nous avons découvert le terme: Lot (jumeau) 580:103. à Yaxchilan. Par exemple dans l'escalier hiéroglyphique 2 marche 7 le texte serait:

\begin{tabular}{|c|c|c|c|}
\hline \multicolumn{4}{|c|}{ 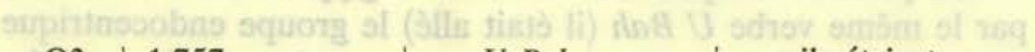 } \\
\hline Q3 & 1.757 . & $U \mathrm{Bah}$ & ils étaient \\
\hline R3 & 16.580: & Yax Lot & $\begin{array}{l}\text { les nouveaux } \\
\text { jumeaux }\end{array}$ \\
\hline Q4 & 1032. & Hun Winic & le $1 \mathrm{er}$ homme \\
\hline R4 & $4.25: 764: 23$. & $\mathrm{NaCan}$ & le 1 er serpent \\
\hline Q5 & III.168:28:548. & Ux Ahaw Katun & $\begin{array}{l}\text { 3ème seigneur } \\
\text { Katun }\end{array}$ \\
\hline R5 & $228.683: 111$ & Ah Winic Bac & $\begin{array}{l}\text { celui aux } 20 \\
\text { captifs }\end{array}$ \\
\hline Q6 & 236.751 . & Yach' Balam & $\begin{array}{l}\text { chef Yach' Balam } \\
\text { (O.J.III) }\end{array}$ \\
\hline R6 & $36.168: 511$. & Ch'uh Ahaw Mul & Divin Seigneur Mul \\
\hline & $36.168: 562$. & Ch'uh Ahaw & Divin Seigneur \\
\hline & & $C h^{\prime} i$ & Chichan \\
\hline
\end{tabular}

A Yaxchilan, plusieurs phrases se référent aux noms des 2 jumeaux 1er homme et 1er Serpent. Le 1er homme est figuré par le guerrier armé d'une lance et d'un bouclier dans la gueule du serpent, scènes plusieurs fois représentées sur les linteaux $13,14,15$ et 25 .

De même dans le vase 117 publié par Robicsek et Hales 1981 sont représentés les jumeaux Hun Ahaw (I.1000f) et Yax Chan (16.1003a). Nous voyons que les noms sont proches de Hun Winic (1032) et $\mathrm{Na}$ Can (4.25.764.23). Dans un autre vase publié par les mêmes auteurs en 1982 p. 41 les 2 jumeaux présentent les glyphes nominaux Hun Ahaw (329.533) et Yax Chan (16.1003a).

Identification des Patrons des scribes et des auteurs des vases

Dorie Reents (1987 p. 125), a identifié le groupe nominal du scribe auteur du texte du vase Grolier 47, confirmé par Stuart (1987 p. 4). De même ce dernier a identifié le nom du peintre du vase publié par Clarkson (1978 fig. 16). Nous pouvons lire ce glyphe 92.168:501. Ahaw Tu-ba glyphe nominal retrouvé à Tikal dans le Temple 4 en L2 en B6. 
D'une façon générale dans la phrase standart le scribe semble être le Seigneur de l'arbre cacao désignant le chef mort (cf. vase Princeton 14) puisque le nom du scribe apparait toujours après le glyphe: $U T z^{\prime} i b$ (son scribe ou il écrit). Le nom d'un scribe particulier peut apparaitre dans une phrase secondaire tel que dans la phrase du Grolier 47.

Lorsque la phrase standart apparait seulement avec le glyphe: $U T z^{\prime} i b$ c'est le cas du Vase 71 publié par Robicsek et Hales 1981 dessous le texte sont représentés 2 jeunes Seigneurs accroupis écrivant sur un Codex, la couverture de bois relevé.

Le patron des scribes semble être le Dieu Singe cf. les vases 63 et 64 des mêmes auteurs malheureusement sans texte. II a été reconnu en premier par Coe 1977.

\section{L'arbre cacao}

La phrase standart contient le glyphe de l'arbre cacao soit de la fente de l'arbuste cacao: Yah Tel Cacaw (Vase Grolier 47) ou du seigneur de l'arbre cacao: Yahaw Te Cacaw (Vase Grolier 49). Chaque chef défunt était associé avec un arbre différent dans le monde souterrain. Ainsi sur les côtes du sarcophage du temple des inscriptions à Palenque sont représentés les ancêtres de Bouclier Pacal enterré dans le sarcophage. Or ces 7 ancêtres sont associés avec 6 arbres différents

$\begin{array}{ll}\text { Cacaw } & \text { (cacaoyer) } \\ Y a & \text { (chicozapote) } \\ T u k & \text { (coyol) } \\ \text { Pachi } & \text { (guayaba) } \\ \text { Haaz } & \text { (mamey) }\end{array}$

et les 4 directions selon Barthel $(1980$, p. 84,85$)$.

Ainsi l'arbre cacao est associé avec le Sud et le Nord et le chef féminin Nah Zac Kuk.

Nous retrouvons dans la phrase standart (vases Princeton 14 et Grolier 47) le titre Nah (premier/mère) associé avec les glyphes de l'arbre cacao. 


\section{Le singe araignée}

La phrase standart se termine souvent par le groupe: Grand enfant originaire du singe araignée: Chac Ch'oc tal maax (cf. vase Princeton 14). Cette expression se rapporte au scribe: Nah Ahaw (le premier seigneur). Sur le plat (116) publié par Robicsek et Hales 1981, nous voyons le jeune Seigneur sortir du sommet du crâne d'un singe et sur le plat 117 . La même scène est accompagnée par les 2 jumeaux Hun Ahaw et Yax Chan. Le jeune seigneur est accompagné de son groupe nominal Hun Ahaw Winic (I.1000f/741 ?) Mais il peut être également le Dieu N dont nous trouvons souvent le glyphe nominal (1014a) suivant le glyphe initial de la phrase standart. Ainsi dans le vase Princeton 19, nous avons lu ce glyphe nominal 1014a; 17 Ah $\mathrm{Pa}-y(i)$ Ah pay (le guide).

Cette interprétation semble confirmé par la scène du vase 56 (cf. Robicsek et Hales 1981). Sur celui-ci le vieux Dieu N est représenté deux fois avec sa coiffure en filet caractéristique ce qui lui donne le nom de Pawah dont la racine: $P a w$ signifie (filet). Le dieu $\mathrm{N}$ semble expliquer le système du compte Maya en s'aidant d'un pinceau et d'un codex à deux autres personnages plus jeunes sans doute des futurs scribes.

D'après Schele et Miller (1986, p. 51), la découverte récente à Copan de la statue d'un scribe accroupi dont la tête à la fois les traits du Dieu $\mathrm{N}$ et du Singe semble illustrer les 3 glyphes de la phrase standart soit le glyphe du Dieu N, le glyphe: $U$ $T z^{\prime} i b$ et celui du Singe araignée Maax.

Nous avons ainsi remarqué que le glyphe: $U T z^{\prime} i b$ peut être remplacé par le glyphe Itzat (artisan) 45.843d. dans les vases Princeton 14 et Grolier 49.

\section{Conclusion}

Dans cette étude sur les vases polychromes Maya nous avons montré que la phrase standart peut être lue phonétiquement ainsi que les phrases secondaires. Ces nouvelles lectures sont dues en partie à un essor d'études des inscriptions des vases mais surtout à la mise au point d'une table syllabique actuellement de 62 valeurs syllabiques notées par près de 369 signes 
que nous venons d'établir à partir des tables syllabiques du groupe d'Albany 1984 et de Stuart 1987.

Elle montre qu'elle peut être utile quelque soit le support des textes glyphiques: Monuments, Codex ou Céramiques.

La phrase standart présente essentiellement le scribe Seigneur de l'arbre cacao enfante du singe araignée. Il est sans doute patronné par le Dieu N: Ah Pay (le guide). Parfois les textes secondaires contiennent les noms des chefs défunts et plus rarement le nom du scribe auteur du vase.

Dans un proche avenir il sera possible d'identifier le nom de ces artistes et de définir les écoles auxquelles ils appartiennent de la même façon que pour les monuments nous pourrons connaitre les noms des sculpteurs qui ont gravé les inscriptions.

VASE PRINCETON 14: JEUNES SEIGNEURS DANSANT AVEC NAIN: Texte cf. Coe 1978 p. 99

\begin{tabular}{|c|c|}
\hline $\begin{array}{l}\text { A } \\
\text { B } \\
\text { C } \\
\text { D } \\
\text { E } \\
\text { F } \\
\text { G } \\
\text { H } \\
\text { I }\end{array}$ &  \\
\hline $\begin{array}{l}\text { J } \\
\text { K } \\
\mathbf{L} \\
\mathbf{M} \\
\mathrm{N}\end{array}$ & $\begin{array}{lll}\text { 1.757b. } & \text { U Bah } & \text { il était allé } \\
12.2 .1041 . & \text { Ah ? Cham ? } & \text { le mort ? } \\
\text { 1010a.184. } & \text { Mah Kina } & \text { tout puissant } \\
126.168: 513 \mathrm{c} . & \text { Yahaw te } & \text { Seigneur de l'arbre } \\
12.671(544): 83 . & \text { Ah Chikinil/Kinichil } & \text { celui de l'ouest/ } \\
& & \begin{array}{l}\text { celui au visage so- } \\
\text { laire }\end{array} \\
229.76 \mathrm{EP}: 81: 102 . & \text { Ah Ch'inil } & \text { celui qui jette } \\
\text { IV.528:200. } & \text { Chan Cup } & \text { les } 4 \text { jicamas } \\
\text { 12.X:501(544).116. Ah Bah Lahkin } & \text { le premier à l'est }\end{array}$ \\
\hline $\begin{array}{l}\mathrm{R} 1 \\
\mathrm{R} 2\end{array}$ & $\begin{array}{l}\text { il était allé } \\
\text { le premier (Seig- }\end{array}$ \\
\hline
\end{tabular}




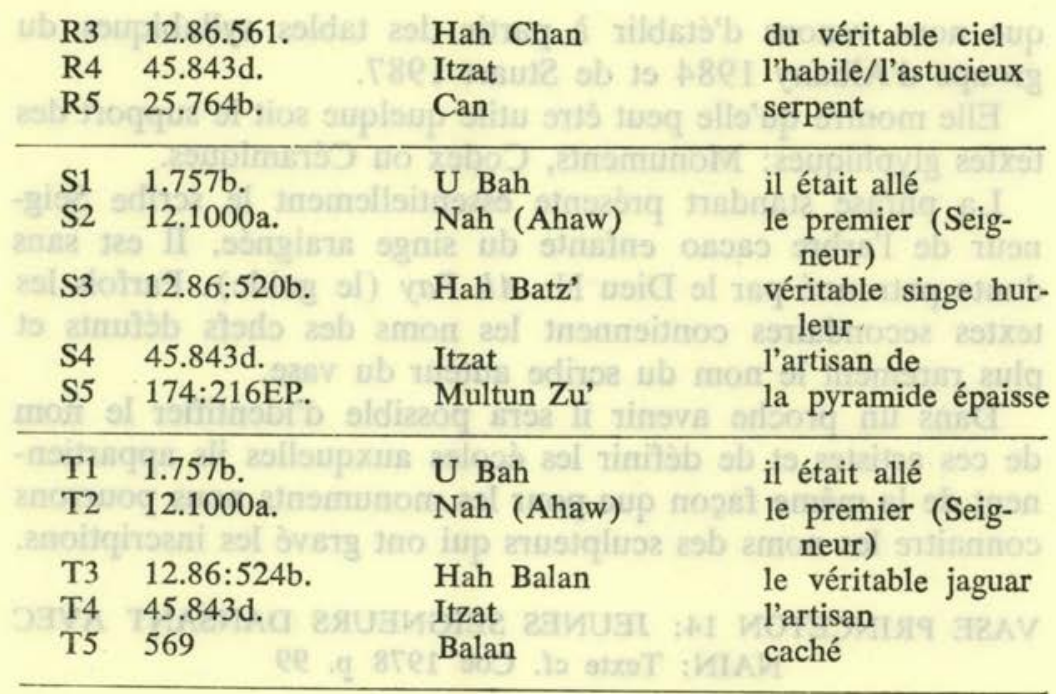

Commentaire du Texte du Vase Princeton 14

Glyphe A: 229.617:126. a-li-(ya) ali avait déclaré

Le préfixe 229 a ici valeur syllabique: $a$, dérivée de: $A h$ préfixe du genre masculin. L'élément principal 617 représente un miroir selon Schele et Miller 1983. Ils lui ont donné la valeur: Nen (miroir). Nous lui avons donnée la valeur Il (voir, regarder) cf. Cordemex p. 267. Ici nous avons donc un glyphe verbal. Sa racine verbale est lue: $A-l(i)$ auquel s'ajoute le suffixe perfectif: $i$ confirmé par le suffixe 126: Ya. Dans le Cordemex p. 9: Aal signifie dire, ordonner, commander. Le suffixe 126: $Y a$, joue le rôle de complément phonétique indiquant la terminaison: $i$ (cf. Stuart 1987 p. 42).

Cette lecture est la plus probable, car dans certains textes un deuxième glyphe transcrit $24: 713 \mathrm{a} .181$ peut suivre ce glyphe initial. Il serait lu: Ilah (avait regardé), l'élément 713a jouant le rôle de piedestal.

Glyphe B: 45.843d. Itz-at Itzat l'artisan

Le préfixe 45 représente une tête de mille-pattes et est un allographe de l'affixe 166 des codex de valeur: Itz dérivée de 
Tzimez (mille-pattes) en Yucatèque. L'élément 843d représente un pénis comme son allographe 761 de valeur: $A t$ (pénis en Chol), d'ou la lecture: Itzat (artisan, astucieux, habile) cf. Cordemex p. 273. Bien que dans ce dernier, il présente la glottale: $T z$ '.

Glyphe C: $\quad 62.77: 585$. u-ch'i-bil(l) Uch'ibil vise

Le préfixe 62 représente un collier de valeur syllabique: $U$ dérivée de $U y$ (collier) d'après Justeson et Campbell (1984 p. 320). Le superfixe 77 représente une aile d'oiseau. Nous lui avons donné la valeur syllabique: Ich'/ch'i dérivée de: Wich' (aile en Chol Palencano) d'après Aulie. Aulie (1978 p. 130). Cette lecture est confirmée par la substitution de l'affixe 77 par l'affixe 236 (oiseau) dans le glyphe transcrit: 62.236:585a, sur un vase de Chochola au Yucatan. Or seulement en Yucatèque: Ch'ich' signifie: Oiseau, d'ou la lecture du glyphe: $U$-ch'i-bi(l) Uch'ibil. Or en Chol Palencano d'après Aulie. Aulie (1978 p. 125). Uch'ibäl désigne la tasse dérivée de $U c h$ ' (boire).

Glyphe D: $\quad 53.61: 565$. $\quad$ ta $u-t(a) \quad T a U t$ était achevé

Le préfixe 53 est lu: $T a$. Le superfixe 61 peut être lu: $U$, comme l'allographe 62: $U$ et l'élément principal 565 est lu syllabiquement: $T a$, d'ou la lecture syllabique: $u-t(a)$. D'après Kaufman et Norman (1984 p. 135), Uht signifie se terminer, s'achever. Ici le glyphe indique que le vase est achevé.

Glyphe E: 1000a. NAH $(A H A W)$ Premier (Seigneur)

La forme anthropomorphe $1000 \mathrm{a}$ est lue: Nah (premier, mère) en Chol. Mais il peut désigner le premier Seigneur: Nah Ahaw indiquant l'un des 3 personnages dansant représenté sous le texte.

Glyphe F: 129:513c.188. Ya(h) te-l(e) Yah Tel fente de l'arbuste

Le superfixe 129 a la valeur syllabique: $Y a$ (allographe du 126), d'après Bricker 1986. Il peut dériver de Yah (fente) (cf. Aulie. Aulie 1978 p. 241). 
L'élément 513c a la valeur: $T e$ (arbre) d'après Stuart (1987 p. 47). Avec le suffixe 188: Le nous avons l'expression: Tel (bois, arbuste) d'après Aulie. Aulie (1978 p. 110). Ces derniers nous ont donné l'expression: I te'el cajpe' (pied ou arbuste de caféier.

Glyphe G: 738c:130. caca-w(a) Cacaw cacao

Cette lecture a été donné par Stuart (1988 p. 154). Ce glyphe désigne le cacao.

Glyphe H: 109.758:110 CHAC Ch'o-c(o) Chac Ch'oc grand enfant

L'elément 758 a été lu: Ch'o par Stuart (1987 p. 46), et le suffixe 110: co par Grube et Stuart 1986. D'ou la lecture Ch'oc (jeune enfant) en Chol d'après Kaufman et Norman (1984 p. 119). Chac a le sens de rouge mais aussi de fort, vigoureux d'après le Cordemex.

Glyphe I: 220d.755 TAL MAAX Tal Maax originaire du singe araignée

Le préfixe 220 d représente une main ouverte pouce dirigé vers la droite. Nous lui avons donné la valeur: Tal (toucher, aller, venir) cf. Cordemex p. 765 . L'élément 755 représente une tête de singe araignée de valeur: Maax d'ou l'expression Tal Maax (originaire, provenant du singe araignée). D'après Robicsek et Hales 1981, dans le vase 116, Hun Ahaw sort du crâne d'un singe. De même le Dieu Singe est le patron des scribes et le Seigneur Hun Ahaw également un scribe, d'après Coe 1977.

Glyphe J: 1.757. UBAH UBah il était allé

L'élément 757 représente une tête de taupe de valeur: Bah cf. Bricker 1986. Mais Bah signifie également premier et fendre en Chol et aller en Tzeltal. 
Glyphe K: 12.?.1041. Ah? CHAM ? Ah Cham ? la mort ?

Le préfixe 12 est lu: $A h$ préfixe du genre masculin. La forme anthropomorphe 1041 représente une tête de mort de valeur: Cham (mort). Malheureusement nous ne pouvons identifier l'autre tête qui entoure celle-ci. Le glyphe désigne peut être le chef défunt. Mais cette lecture reste hypothétique.

Glyphe L: 1010a.184. MAH KINA Mah Kina tout puissant

Ici le titre serait semblable au 74.184. Mah Kina (lecture proposée par Schele 1977). Mais ici le préfixe 74 est remplacé par la forme anthropomorphe du soleil 1010a. Ce titre est retrouvé devant des glyphes nominaux de chefs dans les monuments.

Glyphe M: 126.168:513c. Ya-AHAW te Yahaw te son seigneur de l'arbre

Le préfixe 126 est lu: Ya d'aprés Bricker 1986, p. 64 .

Le superfixe 168 est lu: Ahaw par Justetson et Campbell 1984 et l'élément principal 513c est lu: $T e$ (arbre) soit Ahaw $\mathrm{Te}$ (Son seigneur de l'arbre).

Glyphe N: 12.671(544):83. Ah Chi-KIN-il Ah Chikinil celui de l'ouest

Le préfixe 12 est lu: $A h$ préfixe du genre masculin, comme ses allographes: 228 et 229, l'élément 671 est lu: Chi, l'infixe 544: Kin et le suffixe 83: il soit l'expression: $A h$ Chikinil (celui de l'ouest).

Glyphe O: 229.76EP:81:102. Ah ch'i-ni-l(a) Ah Ch'inil celui qui jette

Le préfixe 12 a la valeur: $A h$ (préfixe du genre masculin).

Le superfixe 76 a la valeur: $C h^{\prime} i$ (cf. notre étude 1987 p. 1522).

Le suffixe 81 a la valeur: $n i$ (cf. notre étude 1987, p. 1524).

Le suffixe 102 a la valeur: $l(a)$. 
L'ensemble serait lu: Ah Ch'i-ni-l(a) Ah Ch'inil.

La racine: $C h$ 'in signifie jeter des pierres, des oranges, des oeufs du mais (conf. Cordemex, p. 137).

\section{Glyphe P: IV.528:200. CHAN cu-p(i) Chan Cup 4 jícamas}

L'élément 528 est lu ici syllabiquement: $c u$ d'après le pseudoalphabet de Landa. Le suffixe 200 est lu: pi selon Stuart 1987, d'ou la lecture syllabique Cup (jícamas) (espèce de tubercule) cf. Cordemex p. 353.

\section{Glyphe $Q: 12 . \mathrm{X}: 501(544) .116$ Ah BAH LAH(un) KIN (ne) $A h$ Bah Lahkin le premier à l'est}

Ici nous avons une forme particulière du glyphe de l'est, mais proche de la forme 546:544.116. En effet ce glyphe est lu: Lah(un) Kin d'après Bricker 1983. Car l'élément 546 est composé du nombre 10: Lahun et de l'élément 544: Kin. Mais ici l'élément 544 est infixé dans l'élément 501 (lis d'eau). Ce dernier serait lu: $B a(h)$ soit $B a h$.
Glyphe R1:
$1.757 b$.
$U B A H$
il était allé

Nous retrouvons la même lecture que pour le glyphe J.

Glyphe R2: $\quad 12.1000 \mathrm{a} \quad \mathrm{NAH}(A H A W) \quad$ Premier (Seigneur)

Nous retrouvons la même lecture que pour le glyphe E.

Ici la forme anthropomorphe 1000a: Nah (Ahaw). Premier (Seigneur) désigne le personnage 2. De plus nous avons un préfixe particulier pouvant se rapprocher de l'affixe 12 (préfixe du genre masculin: $A h$ ).

\section{Glyphe R3: $\quad$ 12.86:561. (a)h-ah CHAN Hah Chan véritable ciel}

Les 2 affixes 12 et 86 , sont lus: (a)h-ah soit $\mathrm{Hah}$ (véritable) en Yucatèque cf. Cordemex p. 168. En effet le superfixe 86 représente le maîs en feuille et a la valeur: $A h$ (nom du jeune 
maîs en Quiché) d'après Edmonson (1965 p. 6). L'élément principal est le glyphe du ciel de valeur: Chan (ciel en Chol).

Glyphe R4: $45.843 \mathrm{~d} \quad$ Itz-at Itzat habile, astucieux

Nous retrouvons la même lecture que pour le glyphe B.

Glyphe R5: $25.764 \mathrm{~b} \quad$ (ca) CAN Can Serpent

L'élément $764 \mathrm{~b}$ représente une tête de serpent de valeur: Chan (serpent en Chol) ou Can (serpent en Yucatèque). Ici le préfixe 25 de valeur: $\mathrm{Ca}$ joue le rôle de complément phonétique indiquant le terme: Can (serpent en Yucatèque).

Glyphe S1:

$1.757 \mathrm{~b}$

$U B A H$

il était allé

Nous retrouvons la même lecture que pour les glyphes J1 et $\mathrm{R} 1$.

Glyphe S2: $12.1000 \mathrm{a} . \quad \mathrm{NAH}(A H A W) \quad$ Premier (Seigneur)

Nous retrouvons la même lecture que pour le glyphe R2.

Glyphe S3: 12.86:520b. (a)h-ah BATZ' Hah Batz' Véritable singe hurleur

Comme pour le glyphe R3, les deux premiers affixes: 12 et 86 sont lus: (a)h-ah soit Hah (véritable). L'élément $520 \mathrm{~b}$ représente la gueule ouverte du singe hurleur d'où la valeur: Batz' (singe hurleur) et également le nom du jour dans les Hautes Terres. A côté du texte le jeune Seigneur (personnage 4) porte sur le dos un dais dans lequel est assis un singe hurleur. En Chol Lacandon et Manché le jour est Batz' d'après notre étude de 1982 p. 20.

Glyphe S4: 45.843d itz-at Itzat l'artisan de

Nous retrouvons la même lecture que pour les glyphes B et R4. 
Glyphe S5: 174:216EP. MULTUN ZU' la pyramide épaisse

Le superfixe 174 est formé de l'élément 528: Tun encadré de 3 points en triangle de chaque côté de valeur: Mul (amonceler) Multun dans le Cordemex p. 540 signifie colline, pyramide. L'affixe 216EP ici utilisé comme élément principal a la valeur syllabique: $z u$ ou la valeur logographique: $Z u^{\prime}$. Dans le Cordemex p. 740, $\mathrm{Zu}$ ' signifie épais, touffu. Ce glyphe est l'élément principal du glyphe emblème de Machaquila.

Glyphe T1: $\quad 1.757 \mathrm{~b}, \quad U B A H \quad U B a h \quad$ il était allé

Nous retrouvons la même lecture que pour les glyphes R1 et S1.

Glyphe T2: 12.1000a. NAH (AHAW) Premier (Seigneur)

Nous retrouvons la même lecture que pour les glyphes R2 et $\mathrm{S} 2$.

Glyphe T3: 12.86:524b (a)h-ah BALAM Hah Balam véritable jaguar

L'élément principal 524b est le glyphe du jour: Hix mais ici sans cartouche il peut avoir la valeur logographique: Balam. A côté du texte le jeune Seigneur (personnage 6) porte sur son dos comme le précédent un dais où est assis un jaguar. A Tikal dans la tombe 116 du Temple 1 l'os M.T.38a porte le groupe glyphique suivant:

\subsection{8:1000a/12.86:524b Ah Nah Ahaw Hah Balam le premier seigneur véritable jaguar}

Ainsi le chef A de Tikal: Ah Cacaw porte un nom identique au 1er seigneur du vase Princeton 14.

Glyphe T4: 45.843d. Itz-at Itzat artisan, habile

Nous retrouvons la même lecture que pour les glyphes R4 et S4. 


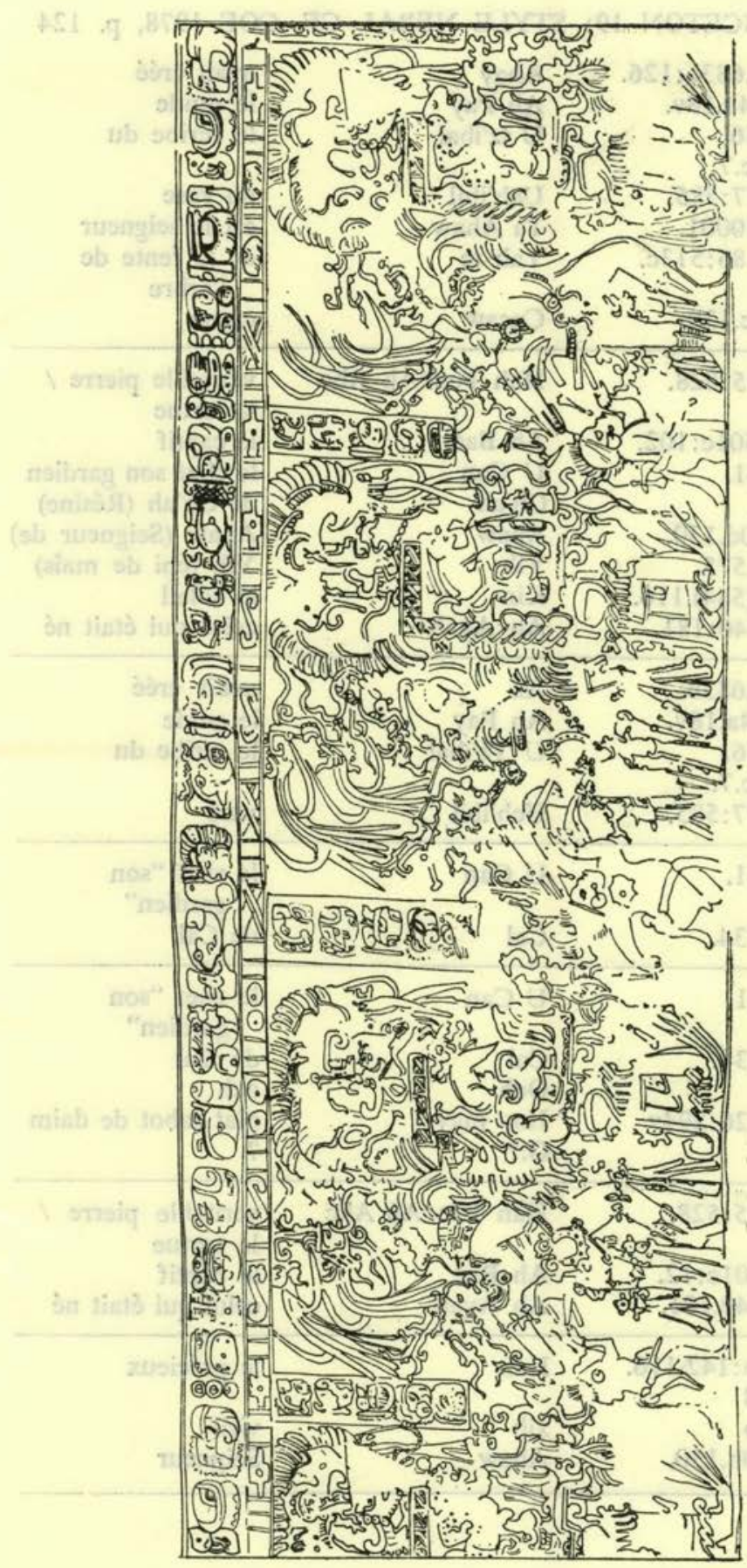


VASE PRINCETON 19: STYLE NEBAJ. CF. COE 1978, p. 124

\begin{tabular}{|c|c|c|c|}
\hline $\begin{array}{l}\text { A } \\
\text { B } \\
\text { C } \\
\text { D } \\
\text { E } \\
\text { F } \\
\text { G }\end{array}$ & $\begin{array}{l}229.683 \mathrm{~b}: 126 . \\
1014 \mathrm{a} .18 \mathrm{v} . \\
1.756 . \\
501 \mathrm{c} .7 . \\
61.77: 585 . \\
51.10001 . \\
126.85: 513 \mathrm{c} . \\
738 \mathrm{c} .130 .\end{array}$ & $\begin{array}{l}\text { Ahay } \\
\text { Ah Pay } \\
\text { U tz'ibal } \\
\text { Uch'ibil } \\
\text { Ta Ahaw } \\
\text { Yah te } \\
\text { Cacaw }\end{array}$ & $\begin{array}{l}\text { avait créé } \\
\text { le guide } \\
\text { le scribe du } \\
\text { du vase } \\
\text { est le seigneur } \\
\text { de la fente de } \\
\text { l'arbre } \\
\text { cacao }\end{array}$ \\
\hline $\begin{array}{l}\mathrm{J} \\
\mathrm{K} \\
\mathrm{L} \\
\mathrm{M} \\
\mathrm{N} \\
\mathrm{O} \\
\mathrm{P}\end{array}$ & $\begin{array}{l}12.85: 528 . \\
12.501 \mathrm{c}: 102 . \\
1.561 \\
603 . \\
1000 \mathrm{~d} .130 . \\
136.575 . \\
756(544) .116 \\
12.740: 181 .\end{array}$ & $\begin{array}{l}\text { Hah Tun/Ah } \\
\text { Ah Bac } \\
\text { U Can } \\
\text { Ch'ah } \\
\text { Ahaw } \\
\text { Yih } \\
\text { Kin } \\
\text { Ah Ayah }\end{array}$ & $\begin{array}{l}\text { véritable pierre / } \\
\text { la tortue } \\
\text { le captif } \\
\text { le chef son gardien } \\
\text { de Ch'ah (Résine) } \\
\text { Ahaw (Seigneur de) } \\
\text { Yih (épi de maîs) } \\
\text { le soleil } \\
\text { celui qui était né }\end{array}$ \\
\hline $\begin{array}{l}\text { Q1 } \\
\text { Q2 } \\
\text { Q3 } \\
\text { Q4 } \\
\text { Q5 }\end{array}$ & $\begin{array}{l}229.683 \mathrm{~b} . \\
1014 \mathrm{a} .18 \mathrm{v} . \\
1.756 . \\
501 \mathrm{c} .7 . \\
61.77: 585\end{array}$ & $\begin{array}{l}\text { Ah } \\
\text { Ah Pay } \\
\text { U Tz'ibal } \\
\text { Uch'ibil }\end{array}$ & $\begin{array}{l}\text { avait créé } \\
\text { le guide } \\
\text { le scribe du } \\
\text { vase }\end{array}$ \\
\hline $\begin{array}{l}\mathrm{R} 1 \\
\mathrm{R} 2\end{array}$ & $\begin{array}{l}1.561 . \\
25.534 .\end{array}$ & $\begin{array}{l}\text { U Can } \\
\text { Cal }\end{array}$ & $\begin{array}{l}\text { le chef "son } \\
\text { gardien" } \\
\text { de Cal }\end{array}$ \\
\hline $\begin{array}{l}\text { S1 } \\
\text { S3 } \\
\text { S4 } \\
\text { S2 } \\
\text { T1 }\end{array}$ & $\begin{array}{l}1.561 . \\
25.534 . \\
790 \\
12.126: 294 \mathrm{v} \\
99 . ?\end{array}$ & $\begin{array}{l}\text { U Can } \\
\text { Cal } \\
\text { Och } \\
\text { Hay may } \\
\text { O.? }\end{array}$ & $\begin{array}{l}\text { le chef "son } \\
\text { gardien" } \\
\text { de Cal } \\
\text { och } \\
\text { plat sabot de daim } \\
\text { ? }\end{array}$ \\
\hline $\begin{array}{l}\mathrm{U} \\
\mathrm{V} 1 \\
\mathrm{~V} 2\end{array}$ & $\begin{array}{l}12.85: 528 \\
12.501 \mathrm{c}: 12 \\
12.740 .181\end{array}$ & $\begin{array}{l}\text { Hah Tun/Ah Ahc } \\
\text { Ah Bac } \\
\text { Ah Ayah }\end{array}$ & $\begin{array}{l}\text { véritable pierre / } \\
\text { la tortue } \\
\text { le captif } \\
\text { celui qui était né }\end{array}$ \\
\hline $\begin{array}{l}\text { W1 } \\
\text { W2 } \\
\text { W3 } \\
\text { W4 }\end{array}$ & $\begin{array}{l}669 \mathrm{~b}: 142.116 . \\
1048 \\
757 \mathrm{~b} \\
1000 \mathrm{~h} .130\end{array}$ & $\begin{array}{l}\text { Kan } \\
\text { xib } \\
\text { Ahaw }\end{array}$ & $\begin{array}{l}\text { le précieux } \\
\text { viril } \\
\text { Seigneur }\end{array}$ \\
\hline
\end{tabular}


$\begin{array}{llll}\text { Glyphe T5: } & 569 & \text { BALAN }\end{array}$

L'élément principal 569 représente un paquet tressé. Il a la valeur Balan (caché) selon Schele (1985 p. 63).

Commentaire du texte du Vase Princeton 19

Glyphe A: 229.683b:126. a-ha-y(a) Ahay avait créé

Le préfixe 229 est lu ici syllabiquement: $a$.

L'élément principal $683 \mathrm{~b}$ est lu: $H a$.

Le suffixe 126 est lu: y(a). L'ensemble serait lu syllabiquement: $a-h a_{-}-y(a)$ soit Ahay (avait crée). Dans le Cordemex p. 3 nous avons: $A h$ (créer, éveiller, réveiller).

Glyphe B: 1014a.18v. Ah pa-y(i) Ah Pay le guide

La forme anthropomorphe 1014 représente la tête du Dieu N. Il peut être lu: Pawah selon Justeson et Campbell (1984 p. 361). Mais il pourrait être lu plus simplement: Paw (filet) et syllabiquement: $p a$, car sur le sommet de la tête il porte un filet. Il est suivi du postfixe $18 \mathrm{v}$ de valeur: $y i$ selon Stuart 1987. Soit la lecture syllabique: $p a-y(i)$ Pay. Dans le Cordemex p. 637 nous avons l'expression: Ah Pay (le guide).

Glyphes $C$ et D: 1.756/501c.7. U Tz'i-ba-l(i) U Tz'ibal Il avait inscrit/son scribe

Le glyphe C représente une tête de chauve-souris (756) de valeur: $T z^{\prime} i$ d'après Stuart 1987 . Le deuxième glyphe comprend l'élément 501c de valeur: $b a$ et le postfixe 7 a la valeur: $l(i)$. Cet affixe 7 est parfois infixé dans la tête de la chauvesouris 756. L'ensemble serait lu: $U t z^{\prime} i-b a-l(i)$ soit $U T z^{\prime} i b a l$ (il avait inscrit). La racine $T z^{\prime} i b$ signifie écrire dans le Cordemex p. 882 .

Glyphe E: $\quad 61.77: 585 \mathrm{a}$. uch'i-bill $U$ Ch'ibil vase

Nous retrouvons le même glyphe dans le vase Princeton 14 en $\mathrm{C}$. 
Glyphe F: $\quad 51.1000 \mathrm{~h} . \quad T a A H A W \quad$ du Seigneur

Le préfixe 51 est un allographe du 59 de même valeur: $T a$ (préfixe locatif).

La forme anthropomorphe $1000 \mathrm{~h}$ a la valeur logographique: Ahaw (Seigneur).

Glyphe G: $\quad 126.85: 513 \mathrm{c}$ Ya-ahte Yah Te la fente de l'arbre

Ici Yah signifie fente cf. Aulie et Aulie (1978 p. 141). Cf. également Vase Princeton 14 en $\mathrm{M}$.

Glyphe H: 738c. 130. CACAW(a) Cacaw cacao

Nous retrouvons le même glyphe dans le vase Princeton 14 en $\mathrm{G}$.

Glyphe I: $\quad 12.85: 528 \mathrm{~b}$.

(a)h-ah TUN Hah Tun véritable pierre $a h-a h-c(u) \quad A h A h c$ la tortue

Deux lectures sont possibles pour ce glyphe. Dans le premier cas, nous lisons les deux affixes: (a)h-ah soit Hah (véritable) et l'élément 528b Tun. Bien que nous n'avons pas ici le complément phonétique: $n i$ (116). Comme l'a remarqué Bricker en 1986 le glyphe du captif: 12.1:528b est lu: $A h U-c(u)$ Ah Uc.

De même le glyphe I pourrait être lu: $A h a h-c(u) A h A h c$ (la tortue).

D'après Kaufman et Norman (1984 p. 115), Ahc désigne la tortue en Proto-Chol.

Glyphe J: 12.501c:102. Ah b a-c(i) Ah Bac le captif

L'élément principal 501c est lu syllabiquement: $b a$ (valeur reconnue par presque tous les auteurs). Il est suivi du suffixe 102: ci soit la lecture: $b a-c(i) B a c$ (os) et par extension: captif. Cette lecture a été donnée par Stuart en 1985. 
Glyphe K: 1.561. UCAAN/CAN son ciel/son gardien

L'élément principal 561 est le glyphe du ciel de valeur: Caan en Yucatèque Houston en 1984 a remarqué l'utilisation du quasi-homonyme: Can (serpent) mais signifiant aussi gardien cf. Cordemex p. 293. En effet, il a remarqué que cet élément peut être remplacé par le glyphe IV: Can ou par la tête de serpent: Can.



Cette lecture a été donnée par Love 1987 pour l'affixe 93. Or il est un allographe de l'élément 603. Il est une partie du nom du captif.

Glyphe M: 1000d.130. AHAW (wa) Ahaw Seigneur

L'élément principal $1000 \mathrm{~d}$ est une tête masculine de profil de valeur: Ahaw (Seigneur). Il est suivi du complément phonétique: $w a$ (130) indiquant bien la lecture logographique: Ahaw (Seigneur) cf. Justeston et Campbell 1984 p. 203.

Glyphe $N$ : $\quad 136.575 \mathrm{v}, \quad y i-h(i) \quad$ Yih épi de maîs

L'élément $575 \mathrm{v}$ est un allographe de l'affixe $17 \mathrm{lu}$ : Y $i$ par Stuart en 1987.

Le préfixe 136 est lu: $h(i)$ d'après le même auteur soit la lecture: $y i-h(i)$ Yih (épi de maîs). Dans le Cordemex p. 976 nous avons l'expression: Yih qui signifie épi de maîs, mais aussi agé, ancien.

Glyphe O: $\quad$ 756(544).116. KIN (ni) Kin Soleil

Ce glyphe est une forme personnifiée de l'élément Kin (544) comme l'élément $765 \mathrm{c}$. Le complément phonétique: $n i$ (116) confirme cette lecture.

Glyphe P:

12.740:181 celui qui était né

Ah $A Y-a h$

Ah Ayah

L'élément principal 740 représente une tête de maiman de valeur: $A y$, signifiant également naitre en Tzeltal d'après Bricker 1986. Il est suivi du suffixe perfectif: $A h(181)$. 
Glyphes $Q 1$ à $Q 5$ :

Ces glyphes sont identiques aux glyphes $\mathrm{A}$ à $\mathrm{E}$.

Glyphe R1: 1.561. $U C A A N / C A N$ son gardien

Il est semblable au glyphe $\mathrm{K}$.

Glyphe R2: 25.534. Ca-l(a) Cal cou

Le préfixe 25 est lu: $c a$ et l'élément 534: $l(a)$. L'ensemble est lu syllabiquement $c a-l(a)$ soit $\mathrm{Cal}$ (cou). Les glyphes R1 et R2 désignent le personnage 1 de Coe 1978 debout tenant un vêtement ou peut-être le personnage 3 agenouillé.

Glyphes S1-T1:

Ces glyphes sont semblables aux glyphes R1-R2.

Glyphe S2: $790 \quad$ OCH Och opossum

L'élément 790 représente une tête d'opossum d'après Thompson 1972, dont le nom est: Och en Yucatèque et Uch en Chol.

Glyphe S3:

$12.126: 294 \mathrm{v}$. Ya-ah $M A Y$ sabot de daim fendu

Yah May

Les deux affixes 126 et 12 sont lus syllabiquement: Ya-ah Yah (fendu) en Chol d'après Aulie. Aulie 1978. L'affixe 294v représente un sabot de daim mais avec les taches noires de la peau de jaguar. Nous aurions peut-être la patte d'animal hybride du daim et du jaguar. Nous avons donné provisoirement la valeur May (sabot de daim).

Glyphe S4: 99 o ?

L'affixe 99 représente une plume de ara de valeur phonétique: $O$. Nous ne savons pas s'il est utilisé comme complément phonétique du glyphe précédent ou comme valeur syllabique propre. 
Les glyphes S1 à S4, désignent le nom du personnage 3 de Coe 1978 agenouillé les bras croisés devant la poitrine avec deux têtes de captif à sa ceinture.

Glyphe U: 12.85:528. (a)h-ah TUN Hah Tun véritable pierre ah $a h-c(u)$ Ah $A h c$ la tortue

Nous retrouvons la même lecture que pour le glyphe I.

Glyphe V1: 12.501c:102 Ah ba-c(i) Ah Bac captif

Nous retrouvons la même lecture que pour le glyphe J.

Glyphe V2:

12.740.181.

Ah Ay-ah

celui qui était né

Ah Ayah

Nous retrouvons la même lecture que pour le glyphe $P$.

Les glyphes U-V2 désignent le nom du personnage 4 de Coe 1978. Le chef principal assis en tailleur sur un trône et le buste penché vers la gauche regarde le personnage 3 qui lui rend hommage.

Glyphe W1: 669b:142.116. Ka-n(i) Kan jaune ou précieux

L'élément principal $669 \mathrm{~b}$ est lu syllabiquement: $k a$, et le postfixe 116: $n i$. Nous avons la lecture: $K a-n(i)$ soit $K a n$ jaune, précieux cf. Cordemex p. 374.

Glyphes W2 et W3: $\quad 1048 / 757 b \quad x i-b(a) \quad X i b \quad$ viril

La forme anthropomorphe 1048 a la valeur syllabique: $x i$ d'après Stuart 1987. Il est lu avec l'élément $757 \mathrm{~b}$ de valeur: $B a$ soit la lecture: $x i-b(a) X i b$ (viril) d'après le Cordemex p. 941. Le glyphe est associé avec le suivant.

Glyphe W4: 1000h.130. AHAW(wa) Ahaw Seigneur

Nous retrouvons la même lecture que pour le glype $M$.

Les glyphes W1-W4 désignent le personnage 5 debout à droite de la scène. 


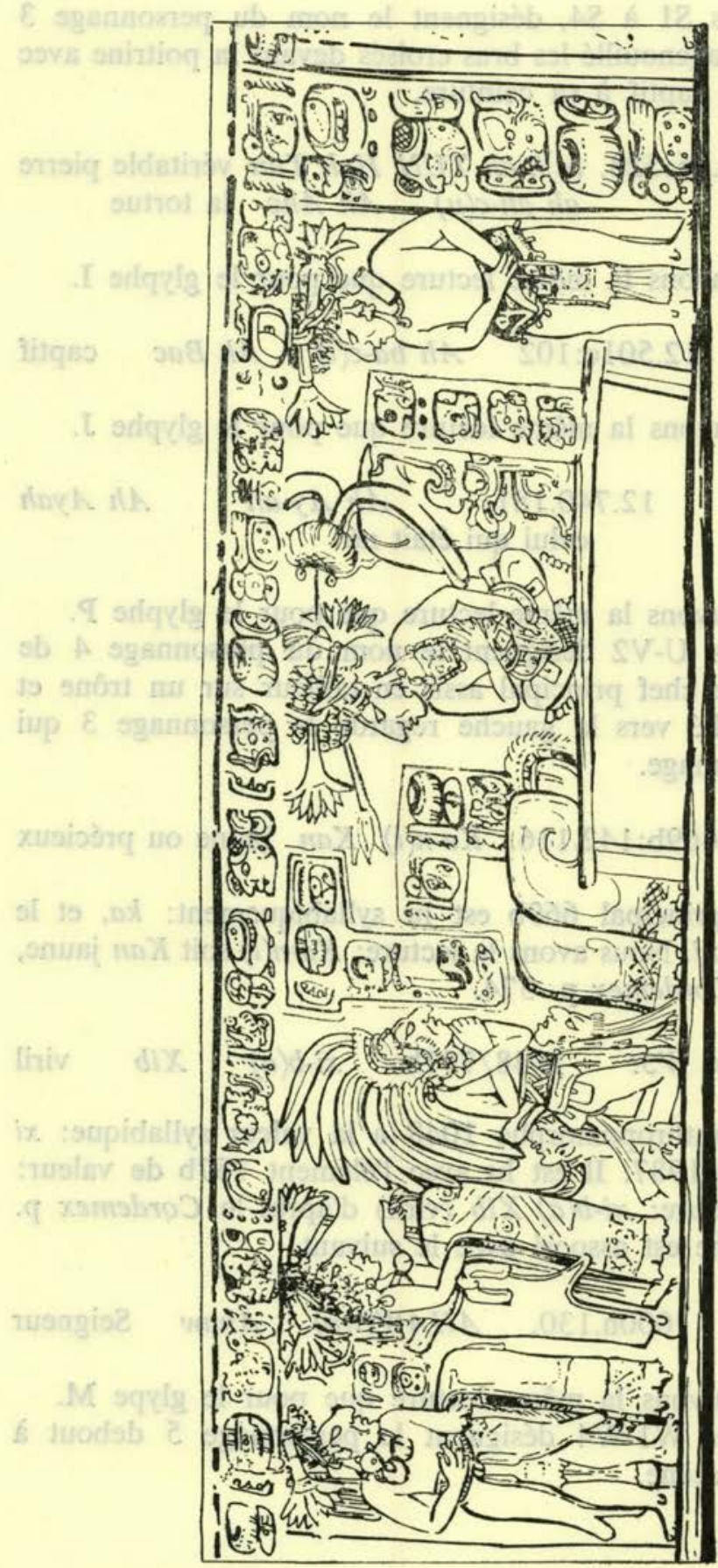


Vase Grolier 47 (cf. COE 1973, p. 103)






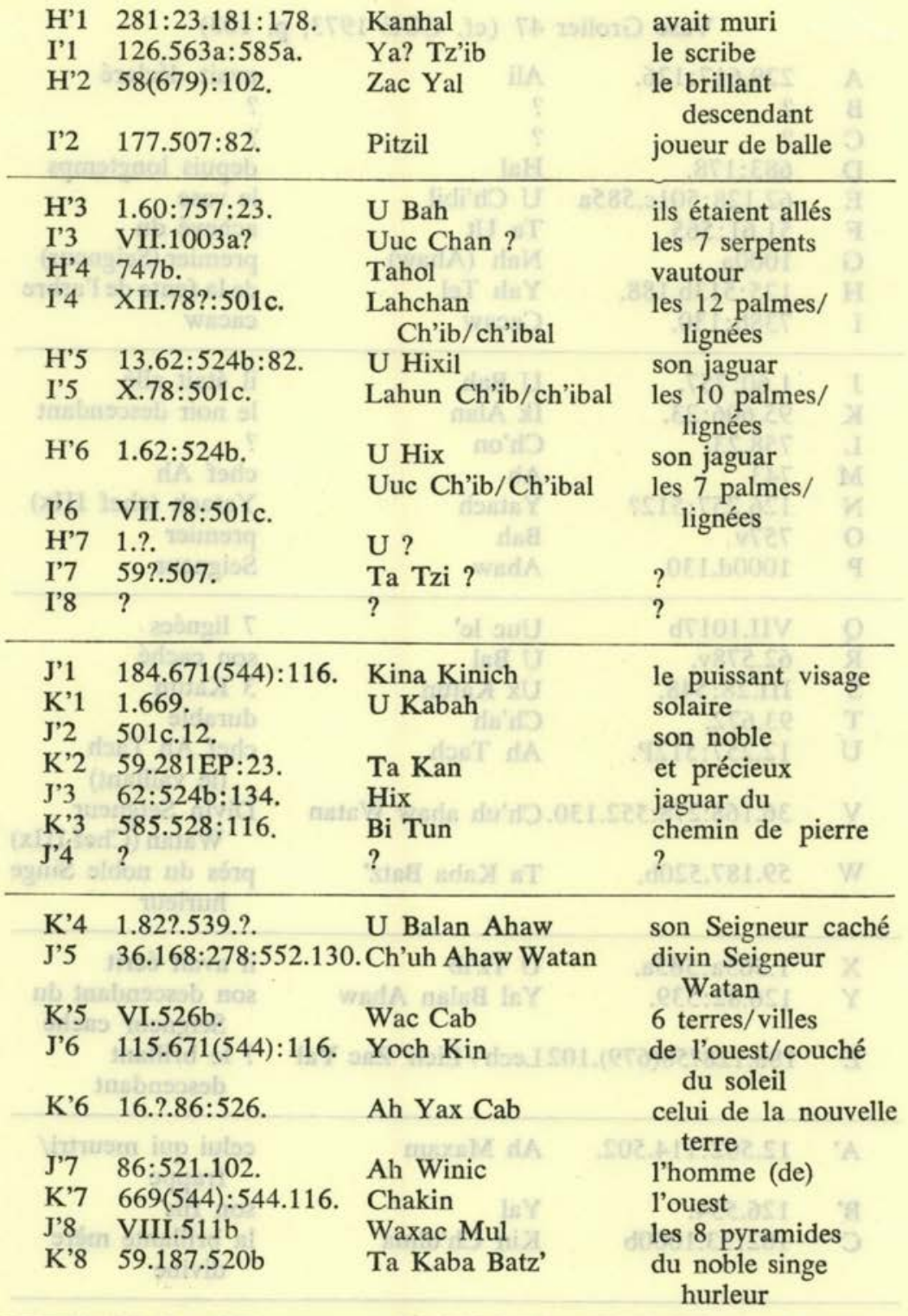









\section{BIBLIOGRAPHIE}

Barrera Vásquez, Alfredo

1980 . Diccionario Maya Cordemex. Maya-Español EspañolMaya, ediciones Cordemex. Mérida, Yucatán, México.

Barthel, Thomas S.

1980 "Mourning and Consolation: Themes of the Palenque Sarcophagus".

Third Palenque Round Table 1978, Part 2, Volume V, pp. 81-90.

University of Texas Press Austin and London.

BRICKER, VICTORIA

1983 "Directional glyphs in maya inscriptions and codices", American Antiquity, vol. 48, no 2, pp. 347-353.

1986. A Grammar of Mayan Hieroglyphs. Middle American Research Institute Publ. 56. Tulane University, New Orleans.

Clarkson, Persis B.

1978 "Classic Maya pictorial ceramics: A Survey of content and theme".

In Papers on the economy and architecture of the ancient Maya, monography VIII, pp. 86-141.

Institute of Archaeology University of California, Los Angeles.

Closs, Michael

1985 "The dynastic history of Naranjo. The Middle Period". In Fifth Palenque Round Table. 1983, vol. VII, pp. 65-77.

Pre-Columbian Art Research Institute, San Francisco.

Coe, Michael

1973. The Maya scribe and his world. The Grolier Club, New York.

1975 Classic Maya pottery at Dumbarton Oaks. Dumbarton Oaks, Washington, USA.

1977 "Supernatural patrons of Maya scribes and artists". In Social Process in Maya Prehistory, pp. 327-347. Academic Press, London.

1978 Lords of the underworld. The Art Museum, Princeton University Press.

1982 Old Gods and Young Heroes. The Pearlman collection of Maya Ceramics. The Israel Museum, Jerusalem. 
Davoust, Michel

1982 Nouvelles Données Historiques dans l'Epigraphie Maya. Thèse de 3ème cycle. Ecole des Hautes Etudes en Sciences Sociales, Paris.

1987 Le Déchiffrement de l'Ecriture Maya: Bilan et Perspectives. Thèse d'Etat.

Ecole des Hautes Etudes en Sciences Sociales de Paris.

EdMonson, MunRo S.

1965 Quiché English Dictionary.

Middle American Research Institute. Tulane University, Publ. 30, New Orleans.

ESCALANTE, ROBERTO

1971 Análisis de Estructuras en el Códice de Dresde.

Grube, Nikolai Cuaderno 4 UNAM, México.

1986 "An investigation of the primary standard sequence on classic Maya ceramics".

Paper presented at the 6th Mesa Redonda de Palenque.

Grube, Nikolai and David Stuart

1987 Observations on T110 as the Syllable ko.

Research reports on ancient maya writing 8.10.

Center for maya research Washington, USA.

Houston, STEPHEN

1987 "Notes on Caracol epigraphy and its significance".

In Investigations At the Classic Maya city of Caracol. Belize: 1985-1987, pp. 85-100.

Pre-Columbian Art Research Institute, Monograph 3.

Houston, Stephen and KarL Taube

1987 "Name Tagging in classic maya script".

Mexicon, vol. 9, no 2, pp. 38-41, Berlin, R.F.A.

Justeston, JoHn S. AND LyLe CAMPBell

1984 Phoneticism in Mayan Hieroglyphic Writing.

Institute for Mesoamerican Studies, State

University of New York at Albany, publ. 9.

Kaufman, Terrence and William Norman

1984 "An outline of Proto-Cholan phonology, morphology and vocabulary", in Phoneticism in Mayan Hieroglyphic Writing, pp. 77-166.

Institute for Mesoamerican Studies, State

University of New York at Albany, publ. 9.

Kelley, David H.

1976 Deciphering the Maya script.

University of Texas Press, Austin and London, USA. 


\section{LOVE, BRUCE}

1987 Glyph T93 and Maya "Hand-scattering" Events. Research Reports on Ancient Maya writing 4 \& 5, pp. 5-16.

Center For Maya Research Washington, USA.

Reents Budet, Dorie

1987 "The discovery of a ceramic artist and royal Patron among the classic Maya", pp. 123-126. Mexicon, vol. IX, no 6, Berlin, R.F.A.

Robicsek, Francis and Donald M. Hales

1981 The Maya book of the dead: The ceramic codex. University of Oklahoma Press, Norman.

1982 Maya ceramic vases from the late classic period. The November Collection of Maya Ceramics. University of Virginia, Charlottesville, Virginia.

SATTERTHWAite, Linton

1963 "Note on hieroglyphs on Bone from the tomb Below Temple 1, Tikal". Expedition, vol. 6, no 1, pp. 18-19. Philadelphia.

SCHele, Linda AND JefFrey Miller

1983 The mirror, the rabbit and the bundle: "Accession" Expressions from the classic Maya inscriptions.

'00nalin Dumbarton Oaks Research Library and Collection. Washington, USA.

SCHELE, LINDA

1979 "Highland Rabbits and Lowland Lords", Actes du 42ème Congrès International des Américanistes. Paris, p. 281-295, vol. 8 .

1985 "Balan-Ahau: A Possible reading of the Tikal Emblem glyph and a title at Palenque", in Fourth Palenque Round Table. 1980, vol. VI, pp. 59-66.

Pre-Columbian Art Research Institute, San Francisco.

Schele, Linda and Mary Ellen Miller

1986 The Blood of Kings: Dynasty and Ritual in Mayan Art.

Kimbell Art Museum Fort Worth.

STUART, David

1985 The Yaxha emblem glyph as Yax-ha.

A New Child-Father Relationship glyph.

Research Reports on Ancient Maya Writing. $1 \& 2$.

Center For Maya Research, Washington, USA. 
1987 Ten Phonetic Syllabes.

Research Reports on Ancient Maya Writing 14.

Center for Maya Research, Washington, USA.

1988 "The Rio Azul cacao pot: epigraphic observations on the function of a Maya ceramic vessel", pp. 153-157. In Antiquity, vol. 62, no. 234, University Press.

THOMPSON J., ERIC

1962 A Catalog of Maya Hieroglyphs. University of Oklahoma Press, Norman.

1972 A Commentary on the Dresden Codex.

Memoirs of the American Philosophical Society 93, Philadelphia. 